\title{
Electrochemical behavior and nanomolar quantification of polydatin using boron doped diamond electrode
}

\author{
Dalibor M. Stanković ${ }^{1,2^{*}}$, Vesna Vukojević ${ }^{2}$, Sladjana Djurdjić ${ }^{2}$, Aleksandar Vukadinović ${ }^{1}$, Miloš \\ Ognjanović ${ }^{1}$, Biljana Dojčinović ${ }^{3}$, Bratislav Antić ${ }^{1}$ \\ ${ }^{1}$ The "Vinča" Institute of Nuclear Sciences, University of Belgrade, POB 522, 11001 Belgrade, Serbia \\ ${ }^{2}$ Innovation Center of the Faculty of Chemistry, University of Belgrade, Studentski trg 12-16, 11000 \\ Belgrade, Serbia \\ ${ }^{3}$ Institute for Chemistry, Technology and Metallurgy, Njegoševa 12, 11000 belgrade Serbia \\ Corresponding author: dalibors@chem.bg.ac.rs daliborstankovic@vin.bg.ac.rs
}

\begin{abstract}
:
In this work we demonstrated electrochemistry and determination of natural compound polydatin and its quantification in tablets. Electrochemical behavior of polydatin was tested in the potential range from -1.5 to $2 \mathrm{~V}$ at unmodified boron doped diamond electrode in various $\mathrm{pH}$ of supporting electrolyte. It has been noticed that polydatin provides two oxidation peaks, one of around 0.73 and second at around $1.3 \mathrm{~V}$. In the reverse scan no reduction peak was observed. Quantification of polydatin was done based on first oxidation peak using square wave voltammetry. After optimization of the method linear working range from 1•10-7 to 7•10-5 $\mathrm{M}$ was obtained, with limit of detection and limit of quantification of $6 \cdot 10-9 \mathrm{M}$ and $2 \cdot 10-8 \mathrm{M}$, respectively. Negligible interferences effect was noted. Developed method shows excellent accuracy and precision toward detection of polydatin and was used for its quantification in the pharmaceutical samples.
\end{abstract}

Key words: polydatin, electrochemistry, boron doped diamond electrode

\section{Introduction}

Polydatin is a natural compound, phenolic type and extracted from the root of the Chinese plant Poligonum cuspidatum. The structural formula of polydatin $(3,4$ ',5-trihydroxystilbene-3- $\beta$-Dglucoside) is shown on Figure 1 . Most often occurs in the grapes skin and presents a precursor of resveratrol which is present in wines and has an antioxidant ability. The biological effect of polydatin is widespread. Namely, a number of studies indicate that polydatin has an antioxidant, anti-cancer, antibacterial, antiallergic, anti-inflammatory effect. In addition, polydatin prevents blood clotting, reduces blood fat levels, but also exposes cardioprotective effect. Also, one study has shown that polydatin is successful during the treatment of chronic pelvic pain associated with endometriosis pain while Xie at all. [1] proved that polydatin has a positive effect in the treatment of diabetes in rats. In addition, the advantages of the electrode are: low value of residual current, minimal noise (high signal/noise ratio), stability in acid and base media, chemical and mechanical stability, as well as as minimal absorption of substances on electrode surface due to presence of sp3 hybridized diamond carbon atom [2-4].

\section{Experimental}

Electrochemical measurements were performed on potentiostat/galvanostat $\mathrm{CHI}$ 760b (USA) utilizing conventional three electrode cell. As a working electrode was used boron doped diamond electrode (BDD, $3 \mathrm{~mm}$ diameter, 1000 ppm of boron doping level, Windsor Scientific, $\mathrm{UK}), 3 \mathrm{M} \mathrm{KCl} \mathrm{Ag/AgCl}$ as reference electrode, while as auxiliary (counter) electrode was used platinum wire of large surface.

\section{Results and discussion}

In the first step, the behavior of polydatin was examined in the potential range of -1.5 to $2 \mathrm{~V}$ (Figure 1). It can be noticed anodic electroactivity of polydatin due to well-defined and sharp oxidation peak at potential of $0.73 \mathrm{~V}$ and second wider oxidation peak at around 1.3 $V$. On other hand, in reversed scan do not occurs reduction peak. Based on this, it can be concluded that oxidation of polydatin in supporting electrolyte irreversible process. Also, it was shown the electrochemical behavior of 
polydatin in positive potential region in BRBS at pH 2 using BDD electrode.

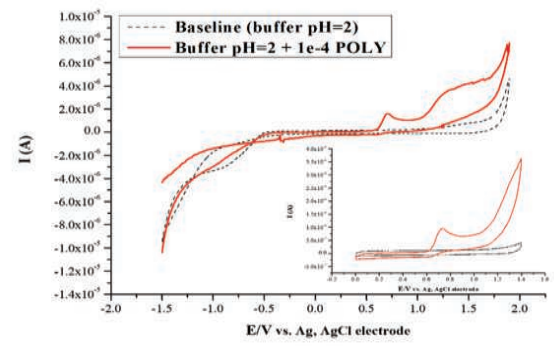

Fig. 1. Cyclic voltammogram of $1 \cdot 10-4 \mathrm{M}$ of polydatin in $B R B S$ at $p H 2$ in fool potential range of $B D D$ electrode. Scan rate $100 \mathrm{mV} / \mathrm{s}$. Insert figure shown electrochemical response of polydatin in positive potential region.Fig. 1 .

Influence of different $\mathrm{pH}$ values on the electrochemical behavior and peak potential and current was studied in the $\mathrm{pH}$ range from 1 to 9. After $\mathrm{pH} 7$ absence of peak was noticed. Based on the obtained results it can be concluded that dominant process which occurs on electrode surface was diffusion controlled. Also, adsorption phenomenon on electrode surface was negligible, or is completely avoided. These statements confirm advantages of selected electrode material and approve idea of this work.

Polydatin in concentration range of $1 \cdot 10^{-7} \mathrm{M}$ to $9 \cdot 10^{-6} \mathrm{M}$ shows one type of process which is played on the BDD electrode, and this dependence can be explained by the equation: $\mathrm{I}(\mathrm{A})=0.06392 \mathrm{C}(\mathrm{M})+7.80524 \cdot 10^{-9}$ were correlation coefficient 0.9977 . The process which is responsible for the oxidation of polydatin on the electrode surface in a low concentration range was diffusion controlled process. Limit of detection was calculated and amounted to $6 \cdot 10^{-9} \mathrm{M}$, while limit of quantification was $2 \cdot 10^{-8} \mathrm{M}$. On the other hand, oxidation peak of polydatin shows also linear dependence in concentration range of $2 \cdot 10^{-5} \mathrm{M}$ to $7 \cdot 10^{-5} \mathrm{M}$, but with another slope, and that calibration curve can be explained by the equation: I $(A)=0.02511 \mathrm{C}(\mathrm{M})+7.13885 \cdot 10^{-7}$, with 0.9961 correlation coefficient. In the area of higher concentrations adsorption phenomenon was responsible for the oxidation of polydatin on the electrode. The optimized SW method was applied for detection of polydatin in POLIDAL tablets.
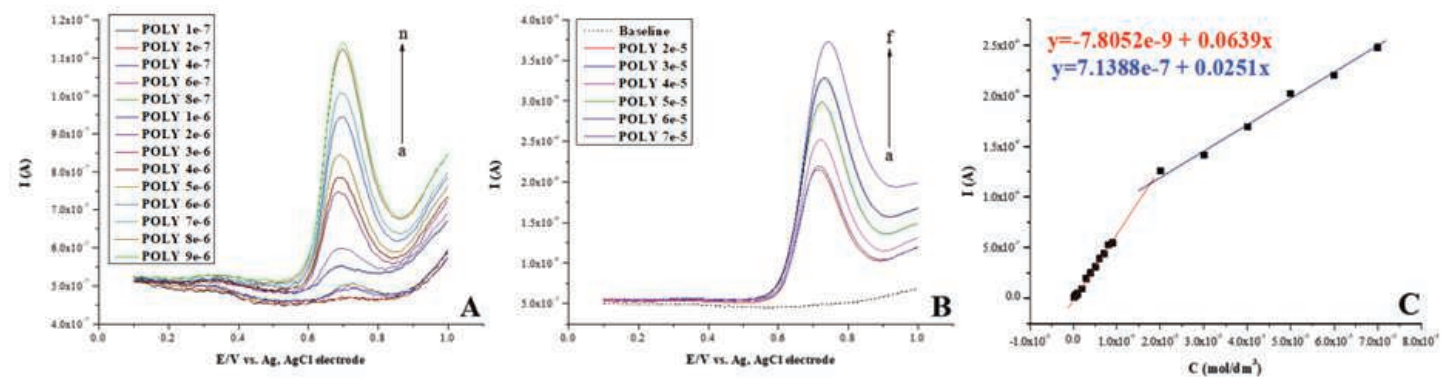

Fig. 2. Voltammograms of dependence oxidation peak of different concentration of polydatin in BRBS pH 2 at $B B D$ electrode; A) from a to $n: 1 \cdot 10-7$ - 9•10-6 M; B) from a to f: 2•10-5 - 7•10-5 M; C) corresponding calibration curves obtained for these measurements.

\section{References}

[1] X. Xie, J. Peng, K. Huang, J. Huang, X. Shen, P. Liu, H. Huang, Polydatin ameliorates experimental diabetes-induced fibronectin through inhibiting the activation of NF-KB signaling pathway in rat glomerular mesangial cells. In Molecular and cellular endocrinology 362 (1-2), 183-193 (2012) doi:.1016/j.mce.2012.06.008.

[2] D.M. Stanković, Sensitive voltammetric determination of thymol in essential oil of Carum copticum seeds using boron-doped diamond electrode, Analytical biochemistry 486 1-4 (2015) doi.org/10.1016/j.ab.2015.06.026
[3] D.M. Stanković, Electroanalytical Approach for Quantification of Pesticide Maneb, Electroanalysis 29 352-357 (2017) doi: 10.1002/elan.201600268

[4] D.M. Stankovi, K. Kalcher, Amperometric quantification of the pesticide ziram at boron doped diamond electrodes using flow injection analysis, Sensors and Actuators B: Chemical 233 (2016) 144-147. doi.org/10.1016/j.snb.2016.04.069 